\title{
Fluoride varnish may be effective in preschoolers
}

\author{
Abstracted from \\ Carvalho DM, Salazar M, Oliveira BH, Coutinho ES. \\ Fluoride varnishes and decrease in caries incidence in preschool children: \\ a systematic review. Rev Bras Epidemiol 2010; 13: 139-149. \\ Address for correspondence: Denise Martins Carvalho. Av. Prefeito Dulcídio Cardoso \\ 3.333/bl.3/ apto. 502 - Barra da Tijuca. Rio de Janeiro - RJ. \\ E-mail: denise_dentist@yahoo.com.br
}

\section{Questions: Does fluoride varnish decrease the incidence of caries in preschool children?}

Data sources BBO, Lilacs, Medline and the Cochrane library were searched.

Study selection Randomised or quasi-randomised controlled clinical trials (RCTs) or controlled clinical trials (CCTs) conducted in children up to six years of age undergoing any form of topical fluoride application reporting caries as an outcome were included. Articles in English, Spanish or Portuguese were included.

Data extraction and synthesis Data were extracted in duplicate and mean caries increment and prevented fraction was presented for each of the included studies. Meta-analysis was not performed owing to the heterogeneity of the data.

Results Eight trials conducted in four countries China, the United States, Poland and Sweden were included. Most of the studies were of poor methodological quality. They were also heterogeneous in relation to participants' previous caries experience, type of intervention administered to the control group, children's exposure to other sources of fluoride and varnish application interval. The absolute differences between caries incidences in the control and test groups ranged from 0.30 to 1.64 and the preventive fractions varied from $5 \%$ to $63 \%$. Conclusions Fluoride varnish may be effective to decrease dental caries incidence in preschoolers, but more randomised clinical trials of better methodological quality are necessary to provide conclusive evidence in this respect.

\section{Commentary}

Dental caries in preschool children is a significant public health problem and largely preventable. Professionally applied fluoride varnish is one of a number of vehicles used for administrating topical fluoride to help prevent or control dental caries. ${ }^{1}$ Fluoride varnish contains high concentrations of fluoride, and a review by Marinho et al. ${ }^{2}$ found that if applied two to four times a year substantially reduces tooth decay in children when used in addition to brushing teeth regularly with fluoride toothpaste. The application of fluoride varnish is recommended in the Department of Health, England's 'Delivering Oral Health-An Evidence-based Toolkit for Prevention' ${ }^{3}$ and is a core component of Scotland's Childsmile programme, a national oral health improvement programme (www.child-smile.org.uk).

The objective of this review was to evaluate the available evidence on the effectiveness of fluoride varnish in decreasing dental caries in children up to the age of six years old. Fluoride varnishes are a convenient method to deliver topical fluoride to preschool children, in a variety of settings, and can take as little as one minute to apply per patient depending on the number of erupted teeth.

The review had a clearly defined question with explicit inclusion and exclusion criteria and the literature search was comprehensive, using clear search terms and included three different languages. The identified studies spanned over thirty years up to December 2008, with no mention of searching the grey literature. The papers were independently reviewed by two researchers, and a table with the excluded studies and reasons for exclusion was supplied.

Eight trials were reviewed, with the majority of them scoring poorly using Jadad's scale to assess the quality of the study. There was limited reporting on the process of randomisation and overall the number of participants at follow up was low.

Due to the heterogeneity of the studies included in the systematic review, meta-analysis was not attempted. There were variations in the participants' previous caries experience, type of intervention administered to the control group, the children's exposure to fluoride and the interval between the varnish applications.

One of the outcome measures was the prevented fraction; this is the difference in caries increments between the treatment and control groups expressed as a percentage of the increment in the control group and ranged from 5 to $63 \%$, with the highest values found in the more recent studies. All studies showed a positive effect and the heterogeneity of the studies meant that an overall estimate could not be calculated. The Cochrane Review ${ }^{2}$ found that fluoride varnish reduced caries by $33 \%$ in the primary dentition, which is within this study's range. Two of the more recent studies in this 
review mentioned that no side effects were reported post application. Marinho ${ }^{2}$ found no information on possible adverse events and none had been reported in more recent studies. ${ }^{1}$

There was no mention in any of the trials about the increased risk of dental fluorosis. A Cochrane review on 'topical fluoride as a cause of dental fluorosis in children' by Wong et al. ${ }^{4}$ looked at the relationship between the use of topical fluorides in children under six years old and the risk of developing dental fluorosis. The majority of the articles in the review concentrated on fluoride toothpaste and fluorosis. It was proposed that future updates would include other fluoride applications if there was relevant new research.

The review was unable to show conclusive evidence of the benefit of fluoride varnish for pre-school children and recommends further robust randomised controlled trials be conducted of sufficient size for this specific age group to provide high quality evidence that is useful when developing policies and oral health programmes. When considering the appropriate time between fluoride varnish applications the review could not make strong recommendations for the ideal interval between fluoride varnish applications but a period of between 3-4 months produced higher prevented fraction results.

\section{Practice points}

- Fluoride varnish should be applied at least twice a year to preschool children's teeth to help reduce dental caries in conjunction with twice daily toothbrushing with fluoride toothpaste and dietary advice.

Emma O'Keefe

Department of Public Health, NHS Fife, Leven, Scotland, UK.

1. Weintraub JA, Ramos-Gomez F, Jue B, et al. Fluoride varnish efficacy in preventing early childhood caries. J Dent Res 2006; 85: 172-176

2. Marinho VCC, Higgins JPT, Logan S, Sheiham A. Fluoride varnishes for preventing dental caries in children and adolescents. Cochrane Database of Systematic Reviews 2002, Issue 3. Art. No. CD002279.

3. Department of Health and British Association for the Study of Community Dentistry. Delivering Better Oral Health: An Evidence-Based Toolkit for Prevention. Gateway Ref:12231 (2009)

4. Wong MCM, Glenny AM, Tsang BWK, Lo ECM, Worthington HV, Marinho VCC. Topical fluoride as a cause of dental fluorosis in children. Cochrane Database of Systematic Reviews 2010, Issue 1. Art. No. CD007693.

Evidence-Based Dentistry (2011) 12, 41-42. doi:10.1038/sj.ebd.6400788 